\title{
An Energy Efficient Clustering Technique in WSNs
}

\author{
Omprakash B, Y P Gowramma
}

\begin{abstract}
Dynamic-The recognition of WSNs has accrued first rate statures once its programs had arrived at various fields like navy. The wireless sensor machine directing conventions can be ordered into degree steerage and numerous leveled or grouping steering counting on their device layout. on this we try to stability the power productiveness at hub degree and expanding the system lifetime through presenting an energy green business enterprise primarily based Hierarchical Routing Scheme. The plan guiding principle of ENEFCT The shape fashionable of ENEFCT is that the manner of Cluster Head have to be circled amongst all hubs conjointly the bunch sizes should be very well determined at very sudden additives of the gadget to weaken vitality

usage and furthermore to extend the gadget life expectancy and electricity electricity.

Watchwords-WSN, ENEFCT, Cluster Head, Cluster vicinity and Cluster Head Nominee
\end{abstract}

\section{INTRODUCTION}

The WSN has an unmistakable component over its mom region remote correspondence advances via larger pursuits in research and further noteworthy progressions in innovation [1][2]. far flung sensor arrange (WSN) are structures which might be profoundly distributive in nature and are progressively self sorted out in usefulness. WSN joins numerous modest sensor hubs in its device, because of this creating a state of affairs that could locate and gather fantastic wonder, it process the detected facts and deliver the results to the bottom station [3]. In WSN the sensor hubs need to organization up amongst themselves as a result of two motives: The mainly else cause is the data this is accumulated from sensor hubs because it offers massive assumptions about nature. the subsequent clarification is that the sensor hubs can provide centered arrangements over correspondence value in opposition to calculation power thru a success coordinated efforts. facts collection is an absolute necessity approach at the same time as sending the information to the base station because the facts got from numerous hubs are profoundly associated therefore the greater records have to be stayed far from within the gadget [4].

\section{ENERGY EFFICIENCY CLUSTERING}

\section{A. institution Formation}

as the makes use of of WSN broadcast a sizable style of fields and vicinity, the control of WSN should be routed to its middle stage; grouping demonstrates to be a a success system

to cope with the sensor hubs along those traces by upgrading its life expectancy. numerous works over group arrangement were proposed with the resource of [5]. The grouping of sensor hubs display up often and the redesign of the framed bunch is carried on a cyclic premise. The originator may be assigned inside the route of the start of every cycle and the messages are communicated the use of distinct strategies that were pointed out in advance than for bunch association. for you to decrease the energy utilization the multi-leap based totally telecom device isemployed.

\section{B. Cluster Head Election}

the subsequent degree as quickly as bunch association is to pick out heads that bypass approximately as pioneer in every group. it is the load of organization heads to combination gaining knowledge of and guidance data from its bunch people to the base station. the burden level of the group head is just like the factors of the bunch. The Cluster head are haphazardly or pre-allotted with the resource of the planner of the gadget.. the choice of bunch head is conveyed discretionarily in arbitrary determination philosophy, the choice is finished bolstered the opportunity that the hub was ne'er constituent as cluster head for the duration of its whole duration within the network. The burden more than one hub to move about as bunch head is in this manner diminished through turning the duty of the huge extensive style of hubs within the institution. The institution head choice is motility and takes vicinity in bound amount of your time. the tactic tries to scale back the energy consumption throughout the communication because it tries to put the cluster head terribly nearly to different nodes within the cluster [6][7].

\section{ClusterOptimization}

the size of the bunch is continuously a large factor that is uncovered to examination in modern machine, at the same time as tending to the issue of energy usage in WSN[8]. For a collection that has littler length the intracluster correspondence would no longer tradeoff a fantastic deal electricity but without a doubt bargain the tool

backbone framework [9]. The tool encounters restrained burdens if the bunch size remains little, but the energy usage is excessive at some level in the intra-employer transmission and the lifetime is pretty decreased. these require an trade off in bunches association. apart from the place of the hub in a set, the dimensions of the institution and its closeness to the base station likewise use the energy resource[10]. The period 


\section{AN ENERGY EFFICIENT CLUSTERING TECHNIQUE IN WSNS}

of the bunch on the underlying structure period of the system consistently live to be arbitrary, besides the facts visitors among the hubs in a set and between the bunches are continuously choppy. Be that as it can, on specific situations the scale of the corporations in the device is probably same which means that the quantities of hubs in all bunches are same[11].

\section{PROPOSED POWER EFFICIENT CLUSTERING}

The method lets in the organization head to talk approximately straightforwardly with the lowest station. The hubs inside the corporation acquaint its top notch and separation with the lowest station by way of the usage of spotting the "welcome" message communicated from the base station at a particular energy diploma at some stage in the device agency degree. The proposed approach contains 3 ranges: I) Cluster head willpower 2) bunch development and three) facts transmission. throughout the organization head dedication approach the model chooses dispersed bunch heads that bears little control overhead. The bunch arrangement technique makes the collection of hubs underneath the organization head. in the ultimate statistics transmission diploma, the bunch head transmits the complete date accumulated from the individuals to the base station as appeared in determine 1 .

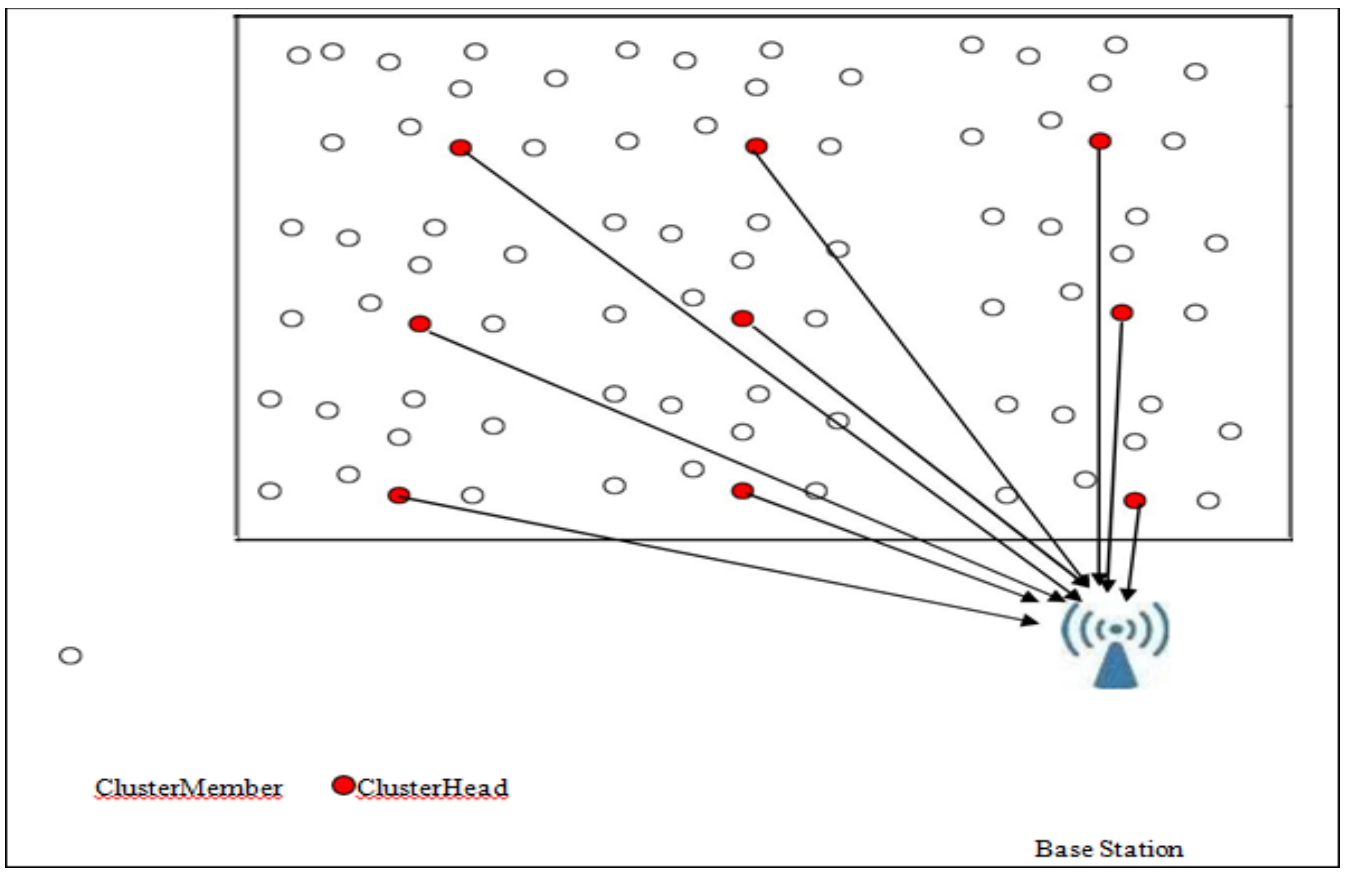

Figure 1 Network Partitioned into Clusters

\section{A. organization Head (CLH) Election}

The duty of the bunch head is rotatedto one-of-a-kind hub within the company once the business enterprise head"s energy stage receives dwindled than that of the regular electricity diploma of the bunch. The bunch head political preference degree segments the device into plenty of Cluster areas (CLR). The hubs which can be accomplishing bunch head desire in each CLR is appointed with a probability scale and then departicipating is known as organization Head Nominee (CLHN). As indicated with the useful resource of this nicely worth, each sensor settles on turning into a Cluster Head Nominee.

particularly, for a hub to turn into a gaggle head it has a probability of $U$, it is scaled through the share of beginning sensor energy degree to the ordinary introductory energy of the device. the next probability for a hub $\mathrm{j}$ in locale $\mathrm{S}$, is given by using

$$
Q_{j}=U * \frac{\text { Residual Node Energy }}{\text { Average Residual Energy }}
$$

(1)

The Qj is processed handiest as quickly as after the gadget instatement. in the beginning, every hub within the institution district chooses an abnormal quantity within the range amongst [0 1]. A sensor hub turns into a Cluster Head Nominee, if the huge range selected is not exactly $\mathrm{Qj}$. alongside those strains, greater or less $\mathrm{T}$ share of hubs inside the bunch locale is chosen as Cluster Head Nominees. while chosen as a Cluster Head Nominee in a Cluster locale CLRj, every Cluster Head Nominee transmits a Cluster Head commercial "CLH ad" package that incorporates the estimation of lingering vitality stage internal a place of positive range rj and it's far decided with the resource of, 


$$
s^{3}=\underbrace{(5)}_{\frac{120^{3}}{\max x}}
$$

The CLHN self assesses with the useful resource of checking the classified ads from different Cluster Head Nominees and it suspends itself from going approximately as a CLH at the off threat that it receives an ad from another CLHN that has higher power diploma than itself. subsequently, the CLHNs that have the most severe closing power among their neighboring CLHNs end up the CLHs. additionally there are probabilities that a Cluster Head Nominee might now not get take a look at parcels for a while, in such instances the CLHN sits tight for Qwait time and after that it's going to consequently change into a Cluster Head hub. This framework encourages in deciding on the actual CLH hubs that depend on the cutting-edge sensor power stocks. similarly, e (CLHN, BAS) art work breaks the tie of Fresidual at some stage in the examinations isused.

\section{STRENGTH CONSUMPTION CALCULATIONS}

\section{Thissectiondiscussesthevalueofenergyconsumptionin}

a Cluster area CLRj during Cluster arrangement, Intra-bunch correspondence and facts preparing and introduces the situation to discern the equal:

\section{A. electricity intake in Cluster Formation}

The Cluster Heads are decided on in a -set up manner and vitality utilization in Cluster Formation as evaluated as pursues. Accepting the length of a control parcel as lo, at that component the absolute grouping energy utilization inside the Cluster area CLRj is calculatedasis

$$
F_{\text {Cluster }}(\mathrm{j})=m x U\left[1_{0}\left(F \sigma_{\mathrm{e}}+\left(\varepsilon f \mathrm{~s} / \Pi \sigma \mathrm{q}_{\mathrm{j}}\right)\right)+\left(\left(\mathrm{U} / \mathrm{q}_{\mathrm{j}}\right)-\right.\right.
$$

$$
\begin{aligned}
& \text { 1) } \left.l_{0} \mathrm{~F}_{\text {ele }}\right]+m x \sigma\left(1-\rho_{\mathrm{j}}\right) \mathrm{l}_{0}\left(\mathrm{~F}_{\text {ele }}+\frac{4 \in f s}{\sigma \pi \tau \sim i^{i}}+\mathrm{F}_{\text {ele }}\right)+ \\
& \operatorname{mx} \sigma \rho_{\mathrm{j}} \mathrm{l}_{0}\left[\left(\mathrm{~F}_{\text {ele }}+\left(\varepsilon f s \alpha^{2} / \Pi \sigma \rho_{\mathrm{j}}\right)+\Pi \mathrm{F}_{\text {ele }}\left(\left(\alpha 2 / \Pi \sigma \rho_{\mathrm{j}}\right) \sigma-1\right]+\mathrm{mx} \quad \sigma\right.\right.
\end{aligned}
$$

$$
\left(\mathrm{F}_{\text {ele }}+\left(\varepsilon f s \alpha^{2} / \Pi \sigma \rho_{\mathrm{j}}\right)+\mathrm{mx} \sigma\left(1-\rho_{\mathrm{j}}\right) \mathrm{l}_{0} \mathrm{~F}_{\text {ele }}\right.
$$

The Equation (3) is explained as pursues. give $\mathrm{Q}$ a chance to be the ratioofall hubs which can be before everything CLH-up-and-comers within the district CLRj, eventually $m \times \sigma Q$ be the amount of opposition in step with region in which $\sigma$ is the hub pressure in the Cluster vicinity. The CHN speaks to the hubs that take care of each different to get selected as a $\mathrm{CH}$ and announce their struggle internal a scope of sweep sj. The CLM hubs get the CLHN productions in every vicinity, that is at the normal of $\Pi s j 2 \sigma \mathrm{U}-1$ sensors percandidate.

in the above circumstance the principle time period speaks to CLHNs assertion about their candidature and getting of these declarations with the aid of way of CLM hubs in the district. within the wake of having chosen, every CLH hub declares its hobby with a CLH-declaration package that is gotten with the resource of those hubs which can be inner its declaration go. the subsequent term speaks to those events. The non-CLH hub in a set district have to ship a manipulate parcel for purchasing related to a $\mathrm{CLH}$, which thusly answers again with an affiliation message, that speaks to the 0.33 term. In conclusion, the final terms are for those CLHs which might be in place $\mathrm{Sj}$ to percentage their time plans a number of the clusternodes.

\section{B. Intra-bunch verbal exchange energy Consumption}

In an intra-bunch correspondence, the sensor hubs which can be individual from a fixed will typify the statistics it noticed in form of a bundle and after that it transmits the parcel to its touching on CLH. Appointing Fbit, because the power predicted to cope with one piece of information, at that factor the strength usage for statistics handling at a CLH within the Cluster region CLRj is alluded as

$$
\operatorname{Fproc}(\mathrm{j})=\operatorname{lm} x \sigma \mathrm{Fbit}(4)
$$

\section{PERFORMANCE EVALUATION \& RESULTS}

The exhibition of the ENEFCT approach has assessed in this segment with the aid of way of using community Simulator 2. The hobby makes use of a similar MAC convention as utilized in LEACH which became obtained in ENEFCT.

\section{A. NetworkLifetime}

lifetime of the gadget is one the parameter to evaluate the usefulness of wireless Sensor Networks. throughout the endeavor, the system lifetime modified into expected by using the use of techniques for man or woman hub cycle i.E., whilst the first Node Dies (FND) and last Node Dies (LND), this is because of the rationale that in statistics social event manner if a hub bites the dust the areas underneath that node" $\mathrm{s}$ inclusion cannot be checked any more.

Framework lifetime (the hour of FND and LND) - to answer how alive a framework is, the which means of framework lifetime may be applied. The framework lifetime may be characterized by way of way of severa methods, it has a tendency to be characterised whilst the fundamental hub bites the dust, or it thoroughly may be characterized via time whilst the final Node Dies, else it could likewise be characterized as even as half of of the hubs kick the bucket or it thoroughly can be

characterized because the time that the machine breaks in as a minimum two sections.

the quantity of hubs alive - finding how many dynamic sensor hubs handy within the whole gadget will keep the ability of detecting and assembling information in a WSN. A dependable WSN is predicated upon on the quantity of dynamic hubs in its situation. therefore, the check of quantity of live hubs inside the tool is applied to assess the usefulness of the WSN framework. The hub consist of which are alive in $100 \mathrm{~m} \times 100 \mathrm{~m}$ machine with a hundred hubs for every 50 rounds became determined. 
desk 1 contrast OF THE network LIFETIME IN ROUNDS

\begin{tabular}{|l|l|r|l|l|}
\hline $\begin{array}{l}\text { Direct } \\
\text { Transmissio } \\
\mathbf{n}\end{array}$ & LEACH & HEED & $\begin{array}{l}\text { ENEFC } \\
\text { T }\end{array}$ & ENEFC \\
\hline 392 & 874 & 1263 & 2065 & T \\
\hline 804 & 1946 & 73 & 3991 & 4799 \\
\hline
\end{tabular}

table 1 delineates that ENEFCT has better consistency time even as contrasted with Direct Transmission, LEACH, HEED and ENEFCTS. The number one hub of ENEFCT is useless after round 2613 rounds at the equal time because the number one hub of Direct Transmission, LEACH, HEED and ENEFCTS is lifeless after round 392, 874, 1263 and 2065 adjusts one after the other as regarded in desk 1 . The device lifestyles of ENEFCT is eighty three.24\%, fifty nine.forty four $\%, 4.70 \%$ and sixteen.eighty three $\%$ better than Direct Transmission, LEACH, HEED and ENEFCTS.

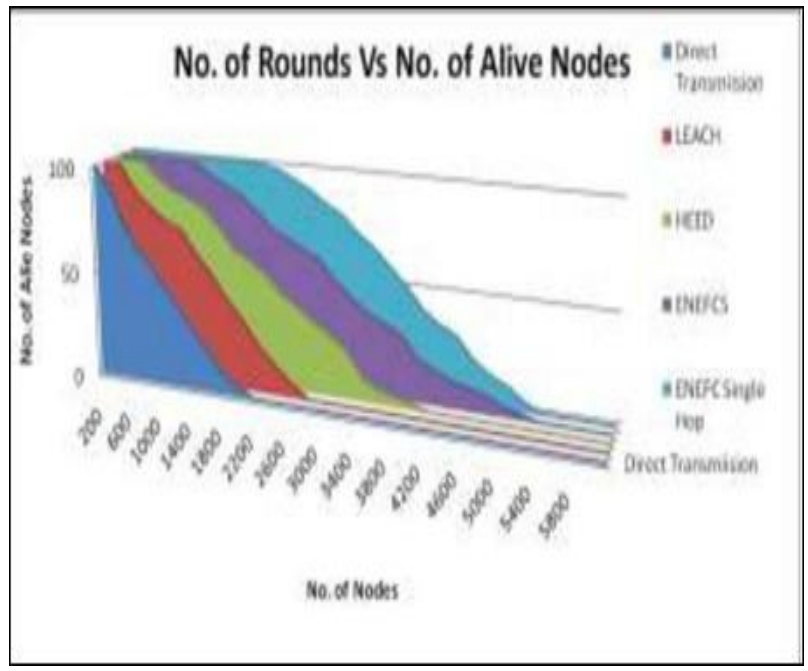

parent 2 contrast of the system lifetime

In determine 2, it emerge as seen that the HEED performs unequalled than LEACH, ENEFCTS perform superior to HEED and ENEFCT performs out the exquisite concerning no. Of rounds. thus it has been presumed that ENEFCT outflanks

both

ENEFCTSandHEED.consequently,ENEFCTaccomplishesw ell

dispersed group heads as indicated thru the device lifetime via adjusting the heap a number of the bunch heads.

\section{B. ResidualEnergy}

it is expected thru ascertaining the combination sum (in joules) of final battery energy toward the end of the exercising. it's miles obvious that ENEFCT hold down appreciably greater strength than Direct Transmission, LEACH, HEED and ENEFCTS. The exercising end end result demonstrates that ENEFCT can boom masses higher electricity productivity than Direct Transmission, LEACH , HEED and ENEFCTS, which protracts the a ways flung sensor community ${ }^{\text {ee }}$ lifetime.

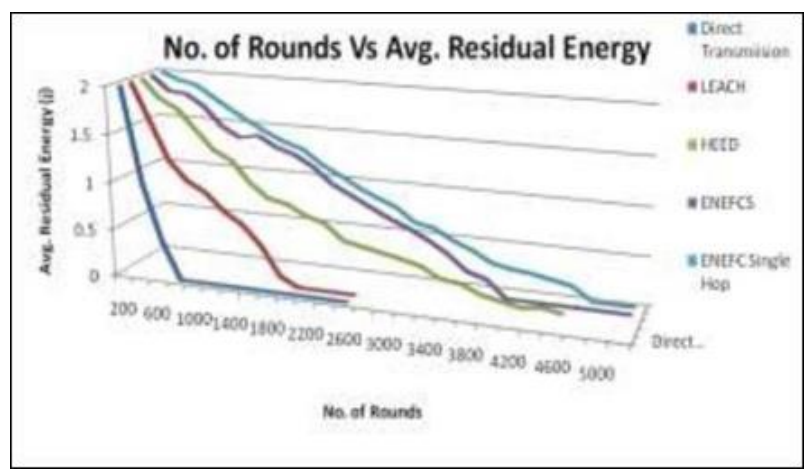

parent 3 impact of ordinary leftover electricity

determine 3 portrays that the leftover power of ENEFCT achieves 0 after around 4610 adjusts however the final power of Direct Transmission, LEACH, HEED and ENEFCTS includes

0 after spherical 684, 1806, 4413, 3802 roundscorrespondingly.

\section{Packet DeliveryRatio}

The parcel conveyance percentage can characterised as the proportion of all out variety of bundles arrived on the reason hub and the whole huge sort of parcels began on the supply hub. The ENEFCT demonstrates 3 - $18 \%$ development in PDR over LEACH, HEED andENEFCTS.

\section{D.Throughput}

The throughput is decided by way of the take a look at of amount of bundles were given on the intention. The result of extensive style of parcels got with assessment in the quantity of hubs. The final results condenses that ENEFCT guarantees about 29.forty one $\%, 37$.Fifty three $\%$, and fifty three.forty four\% extra parcel conveyance to the lowest station as in competition to LEACH, HEED and EECS personally. on this way, the ENEFCT indicates higher throughput when contrasted with LEACH, HEED and ENEFCTS.

\section{END}

This paper, a unique vitality effective and burden adjusted bunching technique ENEFCT has been predicted for periodical statistics accumulating. The pastime results display that the presentation of ENEFCT protracts set up time frame and moreover guarantees night out of hub electricity tiers. On exam with satisfactory in magnificence bunching calculations, as an example, LEACH, HEED and ENEFCTS, the proposed ENEFCT technique performs an lousy lot obviously better as far as power protection and leveling.

\section{REFERENCES}

1. Ahemd, I, Peng, M and Wang, W 2007, „A Unified strength green Bunch identification based Routing Scheme for wi-fi Sensor Networks - An increasingly practical analysisee, 0.33 international conference on Networking and offerings ICNS 2007,PP.86

2. Akkaya. ok, M. Younis, A have a look at on steering conventions for a ways flung sensor systems", ad-hoc Networks, Elsevier.2003

3. Chandrasekaran.V, Shanmugam.A, 2012 "An audit on

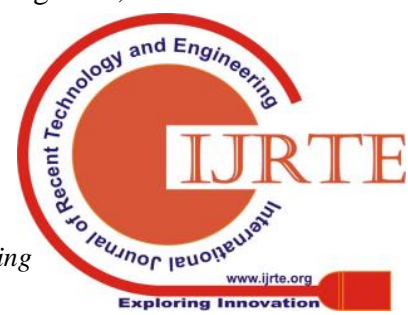


various leveled bunch based directing in wireless sensor systems" journal of worldwide research in computer technological know-how, vol. 3, no. 2,2012 pp.12-16.

4. Akyildiz, IF, Su, W, Sankarasubramaniam, Y \&Cayirci, E 2002, "faraway sensor community: A Survey", computer Networks, Vol. 38, no. four, pp.393-422.

5. Abbasi, AA and Younis, M 2007, „A Survey on Clustering calculations for wi-fi Sensor Networks", computer Communications, Vol. 30, No, 14-15, Pp-2826-2841.

6. Ghiasi, S, Srivatava, A.Yang, X \&Sanafzadeh, M 2002, "best electricity AwareClusteringinClusteringinSensorNetworks",Sensors ,Vol.2 ,pp. 258-269

7. Sohrabi, k,Gao, J, Ailawadhi, V \&pottie,GJ 2000, "conventions for self-business enterprise of a miles off Sensor community", IEEE non-public Correspondences, Vol. 7,No.5, pp.sixteen-27

8. Bandyopadhyay, S and Coyle, EJ 2003, "power green Hierarchical ClusteringAlgorithm for wi-fi Sensor Networks", INFOCOM2003, Vol. 3, PP.1713-1723

9. Sucasas, V.; Radwan, A.; Marques, H.; Rodriguez, J. An assessment on grouping strategies for agreeable far off systems. Impromptu Netw. 2016, 47,53-81.

10. Ahmed, G.; Zou, J.; Zhao, X.; Sadiq Fareed, M.M. Markov Chain version-BasedOptimalClusterHeadsSelectionforWireless SensorNetworks. Sensors 2017, 17, forty four

11. Wang, N.; Zhou, Y.; Xiang, W. An power green Clustering Protocol for Lifetime Maximization in wireless Sensor Networks. In court cases of the 2016 IEEE international Communications conference (GLOBECOM), Washington, DC, united states, 4-eight December2016. 